\title{
Agnès Pivot : l'écriture, processus heuristique et outil pour le dialogue dans une démarche interdisciplinaire
}

\author{
Agnès Pivot ${ }^{\mathrm{a}}$, Nicole Mathieu ${ }^{\mathrm{b}}$ \\ a Sociologue, 20 cité Moynet, 75012 Paris, France \\ b Géographe, UMR Ladyss, CNRS/Université Paris 1, 2 rue Valette, 75005 Paris, France
}

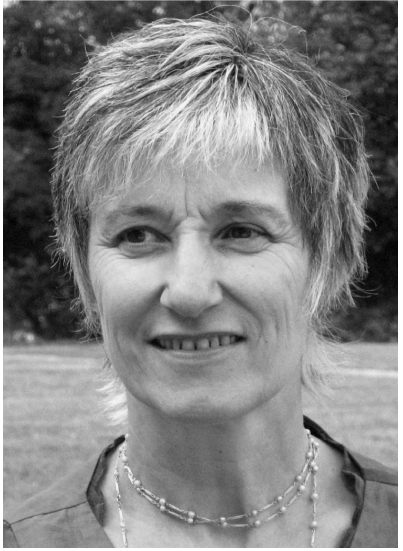

Nicole Mathieu : À l'époque où les programmes Environnement du CNRS étaient à leur apogée, vous avez organisé deux «ateliers d'écriture », l'un pour une équipe interdisciplinaire de l'IFREMER et l'autre pour le PIREN-Seine du CNRS. Aujourd'hui, où, à NSS, on constate toujours la faible place que les articles issus de ces programmes tiennent dans nos colonnes, il me semble important de revenir sur cette expérience dont les leçons pourraient être d'actualité. Mais, d'abord, comment vous est venue cette idée?

Agnès Pivot : L'idée d'ateliers d'écriture était partie d'un constat que nous faisions, Marcel Jollivet et moimême, lorsque que nous étions respectivement rédacteur en chef et rédactrice en chef adjointe de la revue Natures Sciences Sociétés, sur la difficulté d'obtenir des articles émanant des grands programmes interdisciplinaires, alors que la revue était supposée en être un des supports privilégiés.

Mais aussi, et c'est peut-être cela le plus intéressant, d'un autre constat. Vous êtes bien placée, Nicole, pour savoir que les débats du comité de rédaction de NSS sont très animés, en tout cas pour les 10 ans où j'y ai participé. Eh bien, j'avais été frappée par le fait que les articles qui étaient le plus appréciés par nos lecteurs étaient ceux-là mêmes qui avaient occasionné un débat très vif, voire une controverse, au sein du comité et avaient suscité des réactions passionnées. Les discussions tournaient beaucoup autour du récit de la recherche, de son contexte, des enjeux certes scientifiques mais aussi, et il faut bien le dire, des querelles de chapelle et des enjeux de reconnaissance scientifique qui dépassaient le cadre de l'article. Un article avait ses détracteurs mais aussi ses défenseurs, et c'est cette confrontation qui en faisait la richesse. C'est d'ailleurs à cause de cela que nous avons créé les commentaires ainsi que certaines rubriques comme « Regard » ou «Libre opinion », pour publier ces textes qui revêtaient un intérêt certain sans atteindre le niveau dit de rang $\mathrm{A}$, requis pour les articles. Malheureusement, le comité n'ayant lieu qu'une seule journée par mois et avec beaucoup d'articles à examiner, nous n'avions jamais le temps de terminer ces discussions et cela laissait, en tout cas pour certains d'entre nous, un sentiment de frustration. Nous nous disions donc que ce serait vraiment intéressant de pouvoir disposer de temps pour aller jusqu'au fond des choses et que, si nous réussissions à « institutionnaliser » en quelque sorte ces débats, il devrait se passer quelque chose. Étaitce la controverse qui, une fois dépassée, rédigée, se révélait fructueuse? Si oui, comment créer un processus permettant d'arriver à cette formalisation et l'inscrire dans une démarche allant de l'amont à l'aval ? C'est ainsi que j'ai commencé à réfléchir sur l'idée d'un atelier d'écriture et $\mathrm{M}$. Jollivet, alors rédacteur en chef de la revue, m'a suivie avec enthousiasme sur ce projet.

Auteur correspondant : A. Pivot, agnes.pivot1@libertysurf.fr 
N.M. : Je comprends, mais pourquoi l'écriture? Vous êtes sociologue et non littéraire ou linguiste. D'où vous vient cet intérêt pour la langue des scientifiques?

Agnès Pivot : Mon parcours personnel m'avait déjà amenée à m'intéresser à l'écriture et à ses fonctions heuristiques. Au niveau professionnel, avant de prendre mes fonctions comme rédactrice en chef adjointe de la revue, j'avais suivi un stage organisé par le CNRS pour les rédacteurs en chef. J'avais beaucoup apprécié ce stage animé par Jean-Pierre Leguéré, avec qui j'ai voulu collaborer par la suite pour organiser mes propres ateliers. J'avais été frappée de voir quels processus inconscients l'écriture mettait en branle et qu'elle était beaucoup plus qu'un simple acte de mise en forme. En effet, lorsqu'on écrit, on est peut-être seul physiquement avec sa feuille blanche, mais on écrit toujours pour un public, et il est là, très présent dans notre tête. On écrit pour communiquer : de moi à moi, de moi à toi, de moi à eux, de nous à eux. Toutes les combinaisons sont possibles et il y a toujours une dialectique qui s'opère. $C^{\prime}$ est véritablement un processus de communication. C'est alors que je me suis dit que, dans un contexte scientifique, cette fonction pouvait avoir aussi un sens. Car on écrit pour faire son bilan, pour ses pairs, pour être vu, reconnu, pour que notre recherche acquière une visibilité, ce qui est important pour notre carrière. Il y a donc énormément d'enjeux dans cet acte, contrairement à ce que l'on pourrait penser.

J'ai d'ailleurs vu avec plaisir que l'écriture scientifique devient un véritable sujet de recherche et que certains la décrivent comme une « démarche de revendication d'une place quelconque, d'une existence dans un espace social professionnalisé ${ }^{1} »$. Vous imaginez alors si ce n'est pas une action neutre.

N.M. : Pourquoi pensez-vous qu'il soit plus difficile d'écrire un article dans un programme interdisciplinaire?

Agnès Pivot: C'est dû à la vision très spécifique des fondateurs de la revue, pour qui l'interdisciplinarité est une véritable démarche de croisement de disciplines, qui nécessite sur le terrain un dialogue entre elles. C'est celle qui découle de la constatation des limites du pouvoir explicatif $d$ 'une discipline ou d'une spécialité sur certains problèmes de recherche, et donc de l'obligation de construire une pratique de recherche qui assemble de façon méthodique des connaissances, des points de vue, des techniques de travail issues de disciplines différentes. La recherche interdisciplinaire est avant tout une pratique de confrontation des disciplines sur le terrain, une histoire de dialogue, donc, de communication entre gens de disciplines différentes.

1 Bongrand, P., Boursange, É., Déplaude, M.-C., Taiclet, A.-F., 2006. Question à Howard S. Becker. Communication à la journée d'étude Enjeux (et) pratiques de l'écriture en sciences sociales, organisée par l'école doctorale de science politique de Paris 1, Paris 1-Centre Panthéon, Paris, 22-23 septembre.
Mais pourquoi «communication », me direz-vous? La réponse est simple : si vous vous interrogez sur ce qu'est une discipline, vous verrez que c'est une langue habitée par différents cadres de référence, différents types de structure et dont les codes spécifiques entraînent des conventions implicites dont le chercheur n'est plus conscient dans sa pratique quotidienne. Il s'agit de les redécouvrir et de les partager. L'écriture est un des meilleurs moyens de soumettre ces partis pris implicites à l'appréciation de l'autre, puisqu'elle exige la compréhension du texte. Elle laisse des traces derrière elle et, si on peut dire n'importe quoi, on ne peut pas écrire n'importe quoi.

À partir de là, on voit que l'un des obstacles majeurs de cette démarche de terrain est bien la difficulté d'établir une communication efficace entre tous les chercheurs afin de

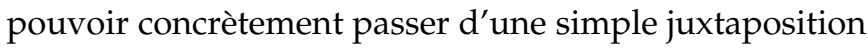
des disciplines à une intégration sans qu'aucune d'elles n'y perde son âme. D'où la justesse des propos de M. Jollivet, entendus lors de l'école thématique d'Aussois ${ }^{2}$ : « L'interdisciplinarité, c'est accepter d'être critiqué par des gens que l'on juge incompétents dans son domaine! » N'estil pas processus plus difficile, plus remuant que cela? Comment le faciliter, le rendre plus interactif, dépasser le simple échange verbal facilement oublié et surtout porteur de malentendus sous-jacents? Comment instaurer ce nécessaire dialogue? Venant d'Angleterre et ayant assisté à de nombreux séminaires résidentiels, j'avais constaté que le temps «hors coulisses", sur quelques jours (les repas pris ensemble, les discussions qui se continuaient au bar, par exemple) pouvait être très instructif et pas du tout inutile. J'avais envie de faire la même chose avec une équipe, afin de tester notre hypothèse qu'il fallait une unité de temps, de lieu et d'action, comme au théâtre. Inutile de vous dire qu'il n'a pas été facile de trouver une équipe qui accepte ce challenge. J'ai organisé cet atelier avec M. Jollivet.

N.M. : Quelle a donc été votre première expérience et quelles sont les principales leçons que vous en avez tirées?

Agnès Pivot: C'est en 1996, en amont d'un programme de recherche d'une équipe venant de l'IFREMER, et je les remercie d'avoir osé une expérience inédite. Le programme qui devait se constituer n'a malheureusement pas pu se développer, mais cela nous a donné l'opportunité de cerner certains des obstacles du dialogue entre chercheurs.

Le premier est celui de l'élaboration d'une problématique commune autour d'un objet de recherche commun. C'est parce que la recherche identifie de nouveaux objets qui ne peuvent être appréhendés par une seule discipline que l'interdisciplinarité se justifie en tant que pratique scientifique et que des chercheurs se donnent un objet

\footnotetext{
${ }^{2}$ La Démarche interdisciplinaire dans le domaine de l'environnement : méthodes et outils pour partager les savoirs, école thématique Ladyss/NSS-Dialogues, Aussois, 29 septembre-2 octobre 2003.
} 
commun. Or, il ne peut être commun que s'il se situe à l'intersection des différents intérêts scientifiques. La définition de cet objet, ses limites et les méthodes propres à le décrire doivent donner lieu à l'élaboration d'un cadre problématique commun à toutes les disciplines concernées.

Comment se poser ensemble les questions essentielles permettant d'identifier les spécificités du terrain, les variables internes aux systèmes étudiés, les variables externes, les unités et les échelles d'observation à privilégier, le prémodèle acceptable pour tous et qui permettra d'orienter une première phase du travail, les représentations conceptuelles et symboliques de l'objet scientifique véhiculées par chaque discipline?

À ce stade, il est fondamental de bien apprécier les différences qui existent entre les cadres de référence des disciplines pour pouvoir identifier la façon de les articuler. $C^{\prime}$ est ainsi que pourra être énoncée une trame de travail en commun, croisant les démarches des uns et des autres et débouchant sur un cahier des charges partagé. Mais tout ceci est plus vite dit que fait.

N.M. : Je vous comprends bien pour avoir vécu ce type d'expériences en tant que chercheur de sciences sociales confronté à des chercheurs de sciences de la vie, mais comment l'écriture peut-elle aider à passer du constat de cette complexité à la pratique?

Agnès Pivot : L'écriture agit comme un révélateur (Encadré), une encre sympathique d'un processus inconscient. Et, comme tout inconscient, elle travaille en soubassement, que vous l'admettiez ou non! En faisant émerger cette dynamique très prégnante au sein de l'équipe, l'écriture permet $d$ 'instaurer le dialogue entre les chercheurs, voire avec les décideurs. Tel biologiste s'apercevra que lorsqu'il parle de ressources à son collègue juriste, il a l'impression de parler du même objet, mais ce n'est qu'une impression. Lorsque les malentendus, les non-dits sont révélés, c'est un facteur très déstressant dans une équipe. Le dialogue peut reprendre sur de vraies bases, saines. Là encore, j'ai eu le plaisir de lire, toujours dans la recherche que je vous ai déjà citée, des propos qui étayent ma réflexion : "Réfléchir à leurs pratiques d'écriture ne ferait pas seulement progresser les chercheurs en sciences sociales sur le plan du style. Ce pourrait être également le moyen de répondre aux angoisses qu'ils éprouvent très fréquemment dans les moments de rédaction et de discussion collective de leurs textes ${ }^{3}$. » Pour moi, ce constat est valable pour toutes les sciences.

De plus, cet atelier, qui a été très dense, très riche, a aussi été, d'après les retours des participants, une source de plaisir non négligeable. Il avait non seulement allégé les angoisses naturelles à un chercheur face à la page blanche, mais les autres l'y avaient aidé, une sorte d'entraide mutuelle s'était établie. Le dialogue était devenu stimulant. En fait, le processus d'écriture est

${ }^{3}$ Bongrand, P., et al., loc. cit. souvent vécu par les chercheurs comme éprouvant, car, consciemment ou inconsciemment, ils en devinent bien les enjeux, que ceux-ci soient intellectuels ou sociaux. Les chercheurs «sentent » bien aussi que les mécanismes physiologiques à l'œuvre dans la lecture (et de facto dans l'écriture) sont complexes. Et ce n'est que récemment que l'on commence à les comprendre. Pour ne donner qu'un exemple, une équipe de chercheurs du CEA a démontré qu'un mot, présenté même brièvement à un sujet, était identifié par le cerveau alors même que la personne affirmait n'avoir rien $\mathrm{vu}^{4}$. Comme le dit si joliment l'intitulé d'un compte rendu de leurs travaux, publié sur le site Internet du $\mathrm{CEA}^{5}$ : «Et si la lecture était tout d'abord un processus inconscient? » On pense que l'on connaît - et vous savez autant que moi que les scientifiques détestent le vide -, mais en fait que maîtrise$\mathrm{t}$-on? Toutefois, on peut au moins prendre conscience de cette complexité et en comprendre un minimum. Démystifier les enjeux rend plus léger. L'écriture comme forme de réduction de l'angoisse du vide, en quelque sorte?

N.M. : Certes, mais pouvez-vous décrire plus précisément les pratiques proprement dites réalisées dans cet atelier?

Agnès Pivot : Lors de l'atelier d'écriture, l'animateur, J.-P. Leguéré, avait, à l'aide d'exercices très simples, démystifié ces processus. La première étape a été de faire comprendre aux chercheurs qu'ils écrivent pour être lus, je le répète, par eux-mêmes, leurs pairs, leurs bailleurs de fonds, etc.

Il avait tout d'abord fait écrire en parallèle à tous les participants une page sur le thème suivant : «Quel est l'apport spécifique de votre discipline au programme de recherche? » Apparemment anodin, cet exercice a permis à chacun de constater, dans un premier temps, comment il se situait dans ce programme - ou, à l'inverse, qu'il ne s'y situait pas! Apprendre comment on lit et surtout comment on interprète ce qu'on lit. Apprendre que le texte que l'on croit lire est, en fait, le résidu du travail qui s'effectue entre le texte lu, la perception, la mémoire de travail et la mémoire profonde. Ces mécanismes de mémoire et leurs précarités sont à connaître, et on constate ainsi que, la plupart du temps, on lit ce qu'on a envie de lire, même dans un contexte scientifique! Pour contrecarrer ces mécanismes inconscients, il faut instaurer une

4 Dehaene, S., Naccache, L., Cohen, L., Le Bihan, D., Mangin, J.-F., Poline, J.-B., Rivière, D., 2001. Cerebral mechanisms of word masking and unconscious repetition priming, Nature Neuroscience, 4, 7, 752-758 (http://www.nature.com/neuro/journal/v4/ n7/pdf/nn0701_752.pdf). Signalons, en outre, la récente sortie d'un ouvrage de S. Dehaene : Les Neurones de la lecture, Paris, Odile Jacob, 2007.

${ }^{5}$ Cf. http://www-dsv.cea.fr/toute-l-actualite/resultats-scientifiques/et-si-la-lecture-etait-tout-d-abord-un-processusinconscient 


\section{Encadré. Les deux étapes principales de l'écrit}

\section{Première étape : l'écrit comme décodeur}

L'écrit permet de :

- garder à l'esprit que l'on s'adresse à un autre que soi ;

- reconnaître les perturbations dans le message que l'on donne et que l'on reçoit (qu'a-t-on vraiment voulu exprimer? Qu'a-t-on lu du message de l'autre?).

Derrière le texte, apparaissent les différents vécus du scientifique : disciplinaire, institutionnel, mais aussi affectif et symbolique. $C^{\prime}$ est pour cela qu'il est intéressant de confronter les écrits des uns et des autres. L'écriture, au démarrage solitaire, et la lecture en commun vont permettre un jeu de miroirs réciproques qui va établir une synergie de base, et les chercheurs pourront ainsi :

- identifier les controverses en les écrivant : c'est dans leur formulation écrite qu'elles prennent une légitimité scientifique et, si le débat s'échauffe, c'est plutôt bon signe;

- visualiser ainsi les tensions entre les codes disciplinaires afin de les travailler (usages différents d'un mot, par exemple) ;

- repérer les différentes représentations conceptuelles et symboliques de l'objet étudié telles qu'elles sont véhiculées par chaque discipline.

\section{Deuxième étape : l'écrit comme catalyseur}

On peut comparer la pratique interdisciplinaire à un jeu de poupées russes, un cadre de référence s'emboitant dans un autre et ainsi de suite. De la fonction de décodage, il est essentiel de passer à une fonction synergique. Pour ce faire, notons plusieurs points intéressants :

- la notion de résultat, si fondamentale par rapport à l'écrit scientifique, est à relativiser en situation d'interdisciplinarité. Nous faisons ici l'hypothèse que c'est le processus du frottement des concepts, ce qu'il en advient à l'issue des échanges, la description de la circularité de la communication, qui constituent le résultat. Par exemple, l'élaboration d'une grille d'analyse peut constituer un résultat en ce qu'elle élucide des présupposés et soit les fait partager, soit les dépasse pour aller vers des propositions de base transversales.

- Le principe de parité entre les disciplines est à respecter. Un vrai processus de décodage suppose l'acceptation de la contestation à l'intérieur du groupe et, donc, d'une certaine remise en question à l'intérieur du groupe et non plus dans sa communauté habituelle.

Forcé d'aller dans des terres inconnues, le chercheur se trouve déstabilisé. Il faut alors qu'il y ait une réassurance, que seul un climat bienveillant peut engendrer. C'est l'élément essentiel que J.-P. Leguéré appelle l'affectio societatis. Sans elle, il n'y a pas de synergie, donc pas de catalyse et pas de recherches!

vigilance, et il existe des techniques pour cela. Il est donc nécessaire de les apprendre. Nous avons pu constater, grâce à ces petits exercices tout simples d'écriture et d'échanges, comment le décodage du langage qui anticipe, accompagne et prolonge la pratique scientifique est un processus négligé par la démarche scientifique, à son détriment d'ailleurs.

J'ouvre ici une parenthèse osée, mais sérieuse : ce serait une bonne chose que les scientifiques, mais surtout les institutions qui les rassemblent, réalisent qu'un chercheur est un homme ou une femme comme les autres, avec des besoins physiologiques, émotionnels et sociaux (de communication, de reconnaissance, de stimulation, etc.) qu'ils amènent aussi avec eux, dans leur équipe, et qu'un travail de réflexion sur le vécu de chacun dans ce contexte n'est jamais redondant. C'est un peu comme un couple (permettez-moi cette comparaison), s'il n'y a pas un effort fait pour une communication efficace, le ronronnement qui s'installe finit par devenir soit explosif, soit amorphe. L'atelier d'écriture est un face-à-face avec soi-même au sein d'un groupe : le syndrome de la page blanche ne concerne pas que l'écrivain et chacun se révèle à travers son texte, même en essayant de ne laisser transparaître aucune passion jugée déplacée.
Lors du deuxième atelier, que j'aborderai plus tard, avec nombre de scientifiques qui n'étaient pas issus des sciences sociales, ceux-ci ont eu beau tenter de se retrancher derrière la neutralité apparente d'une simple écriture de résultats, il était facile d'entrapercevoir certaines caractéristiques de la personnalité de chacun : le bailleur de fonds, grand défenseur du programme, les modélisateurs qui cadrent, ceux qui se réfugient derrière les mots, ceux qui "n'osent pas », les laborieux, les torturés, les affirmatifs. Moi, je trouve cela plutôt touchant, mais fermons la parenthèse avant que votre lecteur n'arrête là !

Pour souligner encore le facteur humain de la science, j'avais été frappée lors du premier atelier par une dimension sur laquelle J.-P. Leguéré avait beaucoup insisté : celle de l'affecto societatis, terme juridique barbare qui recouvre « la volonté de collaborer sur un pied d'égalité à l'œuvre collective ». Si cette volonté n'est pas clairement affichée, avec une équipe qui s'y rallie et accepte un leader qui s'assume en tant que tel, le programme est mal parti.

N.M. : Autrement dit, cette expérience met à nu la part affective de chacun en même temps que la nécessité de la soumettre à une volonté commune que garantit un leader légitime. Mais quel rôle joue l'écriture dans ce processus? 
Agnès Pivot : Cet atelier a aussi donné lieu à une autre piste de réflexion que je n'ai fait qu'ébaucher, à savoir que la collaboration entre les sciences de la nature et les sciences sociales aurait un impact particulièrement bénéfique sur la pratique scientifique. Les sciences sociales ont l'habitude de s'interroger, par l'intermédiaire de la réflexivité, sur les biais inhérents à leurs protocoles. Cela a été rappelé dans le rapport du programme Environnement, vie et sociétés, en 1998, intitulé La Question de l'environnement dans les sciences sociales : « [...] la posture épistémologique des sciences sociales est de prendre de la distance par rapport au sens commun et aux idéologies qu'il véhicule. »

Or, un bon article interdisciplinaire est celui qui met en scène le questionnement, ses raisons, la réflexion généralisée qui en découle; il raconte le récit d'une recherche, et les chercheurs en sciences sociales ne considèrent pas les interrogations sur leur démarche comme des scories à éliminer, mais au contraire comme un enrichissement à leurs recherches. Leur savoir-faire est d'importance dans un programme interdisciplinaire, qui doit nécessairement s'interroger sur ses apports spécifiques.

N.M. : Vous avez mentionné un second atelier? Quelle différence? Quel apport spécifique?

Agnès Pivot: Oui, cette fois-ci en aval de la recherche, avec le PIREN-Seine. L'équipe était réunie pour une tâche monumentale : rédiger une synthèse de quatorze rapports de recherche, qualifiés de «techniques » et couvrant neuf années de recherches. Il s'agissait de faire connaître ces travaux à une communauté scientifique élargie, mais aussi aux bailleurs de fonds et à un public de décideurs et de journalistes scientifiques. L'enjeu était de taille pour le PIREN-Seine, qui voulait ainsi acquérir une meilleure visibilité, mais aussi élaborer un nouveau cahier des charges.

Pour ma part, je me suis moins impliquée dans cet atelier, car son organisation n'était pas véritablement interdisciplinaire au sens où nous l'entendons à NSS, puisque, parmi quarante chercheurs, il n'y avait qu'une représentante des sciences sociales (la géographe humaine Marie-Claude Guerrini, qui d'ailleurs avait une expérience dans le programme Causses-Cévennes que M. Jollivet dirigea dans les années 1980). C'est donc J.-P. Leguéré qui a vraiment pris la responsabilité de cet atelier pour les aider à mener à bien la rédaction de ces rapports pour l'administration. J'en ai tout de même tiré quelques réflexions.

La première, c'est qu'il est plus facile d'instaurer un dialogue entre chercheurs et décideurs en amont d'un programme qu'en aval. En effet, les bailleurs de fonds n'étaient pas tellement intéressés par la démarche mais plutôt par les résultats qui devaient leur permettre de prendre des décisions. Mais, comme ils arrivaient en fin de recherche avec la seule présentation des résultats, ils se trouvaient devant un «fait accompli » et rechignaient à appliquer des mesures qu'ils estimaient bien chères pour des résultats qu'ils jugeaient bien maigres au final (lutte contre l'eutrophisation, par exemple). Cette controverse aurait-elle été évitée dans une élaboration commune en amont de la recherche? J'en suis à peu près convaincue, mais la question reste en suspens, elle nous permet tout de même d'introduire une seconde réflexion.

En ces temps de chamboulement où le «progrès » et ses conséquences, surtout dans le domaine qui nous concerne de la nature, de la science et de la société, sont remis en question (à juste titre d'ailleurs), comment transmettre l'incertitude alors que les décideurs veulent des certitudes pour répondre aux attentes du public? Comment attirer l'attention si on met des fourchettes partout? Comment, donc, parler d'une manière pérenne d'une science toujours en devenir, toujours en construction?

À NSS, nous répondons qu'il faut là s'interroger sur ce qu'est un résultat. Et revenir ainsi à l'écriture, car un résultat, ce n'est pas forcément un fait concret, ce peut être aussi le dévoilement du processus qui a amené à ce fait, qui par définition ne sera un fait «vrai » qu'à un instant $t$. Comme le dit si bien Niels Bohr : «Le contraire de la vérité profonde est une vérité profonde. » Pour ce faire, comme je le disais précédemment, il est nécessaire de mettre le texte en scène : en dévoiler les coulisses, l'éclairage, les jeux de scène, les acteurs, afin de mettre à la disposition des lecteurs l'histoire de la science dont ils sont en train de découvrir les résultats ; cela permet la prise de recul nécessaire.

La troisième réflexion, qui en découle aussi, c'est la nécessité vitale d'avoir du temps pour prendre ce recul. Que de fois n'avons-nous constaté que les chercheurs manquaient de temps pour un travail de réflexion. En faisant écrire leur récit aux participants, nous nous sommes aperçus qu'aucun d'entre eux ne savait vraiment se situer dans le temps et que c'est en tentant de le faire que sont apparues les controverses. Ce phénomène a une explication simple : le nez sur le guidon, les chercheurs n'ont jamais le temps d'ouvrir la réflexion sur leurs résultats - comment y sont-ils arrivés? Pour qui sont-ils écrits? Comment vont-ils être utilisés?

N.M.: Ce sont donc les questions de l'école thématique que vous avez mentionnée : quels liens faites-vous entre cette nouvelle expérience et les précédentes?

Agnès Pivot : En effet, nous étions encouragés par ces deux ateliers à continuer dans la voie de l'instauration d'un dialogue entre chercheurs. Visiblement, nous n'étions pas les seuls à penser cela, puisqu'une commission du CNRS (la 39) a demandé à Yves Luginbühl, alors directeur de l'UMR Ladyss et membre de cette commission, de réfléchir à une école thématique sur l'interdisciplinarité ${ }^{6}$. Au vu de ce que j'avais déjà fait, il m'a chargée de l'organiser.

\footnotetext{
${ }^{6}$ Cf. supra, note 2.
} 
J'étais enchantée car, à NSS, il y a tout un réseau de chercheurs de très grande qualité, de très grande expérience et de très bonne volonté pour échanger avec des chercheurs moins confirmés. Je me réjouissais à l'idée de pouvoir mettre sur pied un tel partage, mais cela ne s'est pas passé exactement comme je l'imaginais. Je voulais faire toute une série d'ateliers réunissant chacun une dizaine - et pas plus - de participants pour qu'ils puissent partager très concrètement leurs expériences. Mais, petit à petit, l'organisation s'est mise aux «normes » scientifiques, puisque nous avons eu de plus en plus $\mathrm{d}$ 'interventions plénières et de moins en moins d'espaces pour la «pratique». Moi y compris, puisque j'ai fait un topo sur ces deux ateliers avec une collègue, Bernadette Leclerc, qui, elle, a parlé d'une expérience très intéressante menée par l'Inra; et devinez où nous l'avons fait... en plénière!

Nous revenons ici au problème mentionné du facteur humain dévalorisé dans la communauté scientifique ; dès que l'on commence à parler dynamique de groupe - car, finalement, c'est bien de cela qu'il s'agit -, une méfiance s'installe. C'est dommage...

J'avais tout de même réussi à ce que la dernière demi-journée soit «libre » et organisée en petits groupes. Là, assez spontanément, un chercheur a eu envie d'appliquer une méthode que je ne connaissais pas mais qui était intéressante, à savoir de travailler sur une question commune et ensuite de permuter entre les groupes. Je leur ai donc demandé de réfléchir sur « leurs attentes et ce qu'ils ont trouvé dans cette école » et de résumer sur un tableau qui pourrait ensuite servir de base commune. Une demi-journée, c'est trop court, d'autant plus que vous savez ce qui se passe en fin de manifestation scientifique, certains participants doivent partir, etc. Mais ce qui est devenu alors évident, $c^{\prime}$ est le besoin de dialoguer qui avait motivé beaucoup de participants pour cette école, et ils se sont donc retrouvés frustrés du manque d'espaces pour discuter, échanger en petits comités, malgré et peut-être surtout à cause de la qualité des interventions et de la participation. Vous pouvez imaginer que ce sont les jeunes chercheurs qui étaient le plus frustrés. Affaire à suivre, donc, peut-être...

N.M. : S'il vous fallait conclure ce "regard", qu'ajouteriez-vous à ces récits de pratiques où l'écriture est un acteur fort?

Agnès Pivot : Je voudrais juste insister sur la fonction synergique de l'accueil «en douceur » des controverses, des conflits sous-jacents. Il est donc important d'avoir des lieux où l'on peut mettre en scène, dans un contexte bienveillant, le dialogue nécessaire à la bonne conduite des recherches interdisciplinaires. J'espère que je vous ai convaincue que, pour moi, la beauté de l'écriture, c'est qu'elle est un catalyseur, qu'elle permet de tisser une trame commune, ce qui ne va jamais sans conflits. L'écriture permet de les mettre au jour et, ainsi, qu'ils ne se transforment pas en mines à retardement. À mon avis, beaucoup de problèmes seraient ainsi évités. Pour moi, l'écriture est définitivement un outil de dialogue utile aux chercheurs et de plus ludique, car une dimension essentielle à l'affecto societatis, c'est aussi une bonne ambiance et donc de l'humour. Je rends hommage à l'équipe de NSS avec qui j'ai travaillé toutes ces années, car elle n'en a jamais manqué! L'humour sauve bien des situations que l'on pense indémêlables (pour ne pas dire autre chose).

Vous voyez, le chantier n'a été qu'entrouvert grâce à ces deux expériences. Elles furent modestes, mais, comme le dit Mère Teresa, l'océan est constitué de petites gouttes d'eau ! Quant à moi, j'y ai eu beaucoup de plaisir et elles m'ont beaucoup appris!

\section{Remerciements}

Je remercie Nicole Mathieu et Marcel Jollivet pour leur approche audacieuse et leur soutien, ainsi que Jacques Quensière de l'IRD pour son apport scientifique lors du premier atelier. Je remercie aussi, pour son inspiration, Jean-Pierre Leguéré, fondateur et directeur de DanaéSciences. Pour plus d'informations, voir le site : http://www.danaesciences.com 